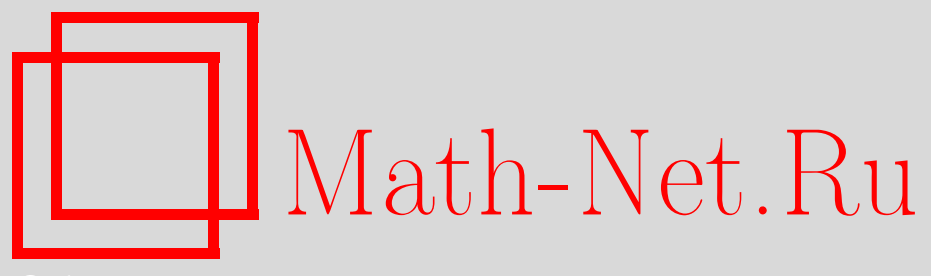

А. М. Райгородский, О дистанционных графах, имеющих большое хроматическое число, но не содержащих больших симплексов, УМН, 2007, том 62, выпуск 6, 187188

DOI: https://doi.org/10.4213/rm7485

Использование Общероссийского математического портала Math-Net.Ru подразумевает, что вы прочитали и согласны с пользовательским соглашением http://www . mathnet.ru/rus/agreement

Параметры загрузки:

IP : 52.23 .180 .231

26 апреля 2023 г., 15:34:58

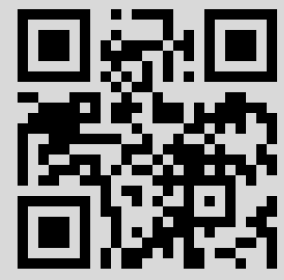




\section{О дистанционных графах, имеющих большое хроматическое число, но не содержащих больших симплексов}

\section{А. М. Райгородский}

1. Введение. В настоящей заметке речь пойдет об одном аспекте классической проблемы Нелсона-Эрдёша-Хадвигера в комбинаторной геометрии. Имеется в виду задача о хроматическом числе пространства $\mathbb{R}^{n}$, т. е. о наименьшем количестве цветов $\chi\left(\mathbb{R}^{n}\right)$, в которые можно так раскрасить все точки пространства, чтобы между одноцветными точками не было расстояния 1 (см. [1]-[3]). В терминах теории графов, $\chi\left(\mathbb{R}^{n}\right)=\chi\left(\mathscr{G}_{n}\right)$, где $\mathscr{G}_{n}=(\mathscr{V}, \mathscr{E})-$ это (бесконечный) граф, у которого $\mathscr{V}=\mathbb{R}^{n}$,

$$
\mathscr{E}=\{(\mathbf{x}, \mathbf{y}) \in \mathscr{V} \times \mathscr{V}:|\mathbf{x}-\mathbf{y}|=1\} .
$$

Здесь через $\chi(G)$ обозначено хроматическое число графа $G$ (см. [4]), а под $|\mathbf{x}-\mathbf{y}|$ мы понимаем (евклидово) расстояние между векторами $\mathbf{x}, \mathbf{y}$. Дистанционным графом называется любой подграф $G$ в графе $\mathscr{G}_{n}$, и сам граф $\mathscr{G}_{n}$ в том числе. Известно, впрочем, что $\chi\left(\mathscr{G}_{n}\right)=\chi(G)$ для некоторого конечного дистанционного графа $G$ (см. [5]), а потому в дальнейшем мы будем фактически говорить лишь о конечных подграфах в $\mathscr{G}_{n}$.

Задача отыскания оценок для $\chi\left(\mathbb{R}^{n}\right)=\max _{G \subset \mathscr{G}_{n}} \chi(G)$ исключительно трудна. До сих пор все, что мы знаем о хроматическом числе плоскости, сводится к неравенствам

$$
4 \leqslant \chi\left(\mathbb{R}^{2}\right) \leqslant 7
$$

(см. [6]), а с ростом размерности зазор увеличивается экспоненциально:

$$
(1.239 \ldots+o(1))^{n} \leqslant \chi\left(\mathbb{R}^{n}\right) \leqslant(3+o(1))^{n}
$$

(cм. [7], [8]).

В серии статей О'Доннелл доказал существование таких дистанционных графов $G$ на плоскости, что $\chi(G)=4$ и в $G$ нет треугольников (см. [9], [10]). Это достаточно яркий результат, ведь априори весьма непросто представить себе, как должен быть устроен граф $G \subset \mathscr{G}_{2}$, у которого максимально большое (среди известных) хроматическое число и который в то же время свободен от полных подграфов на (хотя бы) трех вершинах (четырехвершинных полных подграфов в $\mathscr{G}_{2}$, впрочем, нет по определению).

Аналогичный вопрос естественно поставить и для случая произвольной размерности: существуют ли такие графы $G \subset \mathscr{G}_{n}$, что $\chi(G) \geqslant(1.239 \ldots+o(1))^{n}$, но что, однако же, в $G$ нет симплексов с $\geqslant k$ вершинами? При этом, конечно, $k \leqslant n$, а в идеале $k=3$. В следующем пункте мы сформулируем новые теоремы, в которых дается частичный ответ на поставленный вопрос. Кроме того, мы скажем несколько слов о доказательствах теорем.

\section{2. Формулировки результатов и наброски их доказательств.}

Tеорема 1. Существует такой граф $G \subset \mathscr{G}_{n}$, что $\chi(G) \geqslant(1.239 \ldots+o(1))^{n}$ и в $G$ нет симплексов $c k \geqslant c n$ вершинами, где $c-$ абсолютная константа, меньшая единицы.

Teорема 2. Cуществует такой граф $G \subset \mathscr{G}_{n}$, что $\chi(G) \geqslant(c+o(1))^{n}$ и в $G$ нет симплексов $c k \geqslant f(n)$ вершинами, где $c$ - абсолютная константа, большая единицы, $a f(n)=o(n)$.

Работа выполнена при поддержке гранта РФФИ № 06-01-00383, гранта Президента РФ МД-4235.2006.1, гранта НШ-1312.2006.1, гранта ИНТАС 03-51-5070, гранта фонда “Династия". 
Преимущество теоремы 1 в том, что граф, существование которого в ней утверждается, имеет (асимптотически) самое большое известное хроматическое число. Что же до теоремы 2 , то в ней мы сталкиваемся, по сути, с не слишком значительной потерей в величине $\chi(G)$; при этом, однако, понижение максимальной размерности симплекса, содержащегося в $G$, весьма ощутимо.

Для доказательства теоремы 1 достаточно взять граф $G=(V, E) \subset \mathscr{G}_{n} \mathrm{c}$

$$
\begin{gathered}
V=\left\{\mathbf{x}=\left(x_{1}, \ldots, x_{n}\right): x_{i} \in\{-t, 0, t\} \quad \forall i,\left|\left\{i: x_{i}=t\right\}\right|=a,\left|\left\{i: x_{i}=-t\right\}\right|=b\right\}, \\
E=\left.\mathscr{E}\right|_{V},
\end{gathered}
$$

где $a=[0.36 \ldots \cdot n], b=[0.06 \ldots \cdot n]$ (см. [7]) и величина $t$ выбирается так же, как ее аналог в основной теореме из статьи [7]. Рассматривая этот граф, получаем оценку $\chi\left(\mathbb{R}^{n}\right) \geqslant(1.239 \ldots+o(1))^{n}$, и нетрудно показать, что $\omega(G) \leqslant c n, c<1(\omega(G)-$ это размер самой большой клики в графе $G$, т. е. число вершин в самом большом симплексе, который содержится в $G$, см. [4]).

Доказательство теоремы 2 значительно тоньше. Оно осуществляется с помощью сочетания линейно-алгебраического и вероятностного методов в комбинаторике (см. [11], [12]). Грубо говоря, сперва на графе $G$, описанном выше, или на некотором его аналоге вводится структура, подобная структуре случайного графа в модели $G(m, p)$ (см. [12], [13]). А затем производится достаточно аккуратный анализ того, как много ребер может быть на данном (любом) множестве вершин $W \subset V$, и в результате устанавливается тот факт, что "почти все" подграфы $H$ графа $G$ имеют экспоненциально большие хроматические числа и в то же время для каждого из них $\omega(H)=o(n)$.

Отметим, что доказательство теоремы 2 совершенно неконструктивно и было бы, безусловно, очень интересно получить “эффективизацию” этой теоремы (хотя бы даже ослабив условие экспоненциальности хроматического числа). Также интерес представляет задача построения дистанционных графов с достаточно большим хроматическим и малым "кликовым" числами и с достаточно малым количеством вершин. У графа $G$, о котором шла речь выше, количество вершин тоже экспоненциально.

\section{Список литературы}

[1] А. Сойфер, Математическое просвещение, третья сер., 8, МЦНМО, М., 2004, 185-221; http://www.mccme.ru/free-books/matpros9.html. [2] А. М. Райгородский, УMH, 56:1 (2001), 107-146; англ. пер.: А. M. Raigorodskii, Russian Math. Surveys, 56:1 (2001), 103-139. [3] L. A. Székely, Paul Erdôs and his mathematics, II (Budapest, 1999), Bolyai Soc. Math. Stud., 11, János Bolyai Math. Soc., Budapest, 2002, 649-666. [4] Ф. Харари, Теория графов, Мир, М., 1973; пер. с англ.: F. Harary, Graph theory, Addison-Wesley, Reading, MA-Menlo Park, CA-London, 1969. [5] N. G. de Bruijn, P. Erdős, Nederl. Akad. Wetensch. Proc. Ser. A, 54 (1951), 369-373; Indag. Math., 13 (1951), 371-373. [6] H. Hadwiger, Elem. Math., 16 (1961), 103-104. [7] А. М. Райгородский, УМH, 55:2 (2000), 147-148; англ. пер.: А. M. Raigorodskii, Russian Math. Surveys, 55:2 (2000), 351-352. [8] D. G. Larman, C. A. Rogers, Mathematika, 19 (1972), 1-24. [9] P. O'Donnell, Geombinatorics, 4:1 (1994), 23-29. [10] O'Donnell, Geombinatorics, 5:1 (1995), 31-34. [11] А. М. Райгородский, Линейно-алгебрачческий метод в комбинаторике, МЦНМО, М., 2007. [12] N. Alon, J.H. Spencer, The probabilistic method, 2nd ed., Wiley-Intersci. Ser. Discrete Math. Optim., Wiley-Intersci., New York, 2000. [13] B. Bollobás, Random graphs, 2nd ed., Cambridge Stud. Adv. Math., 73, Cambridge Univ. Press, Cambridge, 2001.

A. М. Райгородский (А. M. Raigorodskii)

Московский государственный университет

им. М. В. Ломоносова

E-mail: araigor@avangard.ru
Представлено В. М. Тихомировым Принято редколлегией 27.07.2007 